\title{
Not All "BAD" Cholesterol Carriers Are Necessarily Bad and Not All “G00D” Cholesterol Carriers Are as Good as Can Be: Plasma Delipidation, a Non-Pharmacological Treatment for Atherosclerosis
}

\author{
Bill Cham \\ Australasian Medical Research, Port Vila, Republic of Vanuatu \\ Email: bill.cham@gmail.com
}

Received 20 July 2015; accepted 21 September 2015; published 24 September 2015

Copyright (C) 2015 by author and Scientific Research Publishing Inc.

This work is licensed under the Creative Commons Attribution International License (CC BY).

http://creativecommons.org/licenses/by/4.0/

(c) (i) Open Access

\begin{abstract}
More than four decades ago it was established that an elevated low-density lipoprotein-cholesterol level was a risk for developing coronary artery disease. For the last two decades, statins have been the cornerstone of reducing low-density lipoprotein-cholesterol, but despite significant clinical efficacy in the majority of patients, a large number of patients suffer from side effects and cannot tolerate the required statin dose to reach their recommended low-density lipoproteincholesterol goals. Preliminary clinical studies indicate that monoclonal antibodies to PCSK9 appear to be highly efficacious in lowering low-density lipoprotein-cholesterol with a favourable adverse event profile. However, further longer-term clinical studies are required to determine their safety. From the early-proposed concept for high-density lipoprotein-mediated cholesterol efflux for the treatment of coronary artery disease, the concentration of the cholesterol content in high-density lipoprotein particles has been considered a surrogate measurement for the efficacy of the reverse cholesterol transport process. However, unlike the beneficial effects of the statins and monoclonal antibodies to PCSK9 in reducing low-density lipoprotein-cholesterol, no significant advances have been made to increase the levels of high-density lipoprotein-cholesterol. Here it is shown that by a non-pharmacological plasma delipidation means, the atherogenic low-density lipoproteins can be converted to anti-atherogenic particles and that the high-density lipoproteins are converted to particles with extreme high affinity to cause rapid regression of atherosclerosis.
\end{abstract}

\section{Keywords}

Plasma Delipidation, Lipid Apheresis, Regression, Atherosclerosis, Pre- $\beta$ HDL

How to cite this paper: Cham, B. (2015) Not All “BAD" Cholesterol Carriers Are Necessarily Bad and Not All "GOOD" Cholesterol Carriers Are as Good as Can Be: Plasma Delipidation, a Non-Pharmacological Treatment for Atherosclerosis. International Journal of Clinical Medicine, 6, 690-699. http://dx.doi.org/10.4236/ijcm.2015.69092 


\section{Introduction}

\subsection{Early Observations of Lipoproteins and Their Relationships with Atherosclerosis}

More than 40 years ago, the Framingham Heart Study established that an elevated low-density lipoprotein-cholesterol (LDL-C) level was a risk for developing coronary artery disease (CAD) [1].

Also, more than 40 years ago, Glomset [2] proposed that high-density lipoprotein (HDL) transfers cholesterol from peripheral tissues to the liver, where metabolites of the sterol are excreted into the bile. From the early proposed concept for HDL-mediated cholesterol efflux [3], the concentration of the cholesterol content in HDL particles has been considered a surrogate measurement for the efficiency of the reverse cholesterol transport (RCT) process.

In the 1970s it was well established that plasma contained lipid carriers, some of which were detrimental to the body, LDLs, and others that were beneficial to the body, HDLs.

\subsection{Atherosclerosis Formation}

Lipoproteins carry cholesterol to and from the vessel wall; if too much cholesterol is carried to and too little is carried from the vessel, it accumulates there and causes fatty deposits to build up in the vessel wall, a phenomenon known as atherosclerosis.

Atherosclerosis, the horror of mankind, refers to the build-up of cholesterol, other fats, and other substances such as calcium deposits, cells, cell debris in, and on, artery walls (plaques), which can restrict blood flow.

Blood clots can form at the plaque and either obstruct blood flow, or, break off and clog the arteries such as the coronary arteries to the heart or carotid arteries to the brain, resulting in a possible heart attack or stroke respectively.

During the pathogenesis of atherosclerosis, blood monocytes, originated from bone marrow-derived progenitor cells, infiltrate from blood to the intima and sub intima, a process that is activated by subendothelial accumulation of apolipoprotein B-containing lipoproteins (LDLs).

The accumulation of apolipoprotein B-lipoproteins (Apo B-LPs) in the matrix beneath the endothelial cell layer leads to the recruitment of monocytes. These cells differentiate into macrophages and undergo foam cell formation.

Macrophage scavenger receptor (SR) binds and internalise modified forms of LDL through mechanisms that are not inhibited by cellular cholesterol content and they are likely responsible for macrophage cholesterol accumulation.

\subsection{Prevention of Atherosclerosis}

Over the past decade, statins, with maintaining a healthy lifestyle, have been the most effective therapies in treating atherosclerosis by reducing the synthesis and thus delivery of cholesterol in Apo B-LPs to the vessel wall. This has resulted in decreased incidences of heart disease by 30 per cent.

There is, however, an urgent need for further reduction of the unacceptably high remaining risk of cardiovascular and cerebrovascular diseases.

Coronary heart disease (CHD) and stroke, caused by atherosclerosis, are leading causes of death in the USA. More than 80 million Americans, one in three of the population, suffer from atherosclerotic disease; most don't even know it. More than 2600 Americans die every day because of cardiovascular diseases, approximately 1 death every 33 seconds.

In essence, drugs like the statins can be regarded as preventative measures of atherosclerosis, that is, statins may inhibit the progression of the disease, which on its own is a major achievement. However, there are no convincing data to show that the statins can cause regression of atherosclerosis.

The monoclonal antibodies alirocumab and evolocumab that block the gene PCSK9 show promise to reduce LDL-C even more effectively than statins. The need to combat atherosclerosis is recognized worldwide. Consequently, health authorities are being forced to approve these promising new drugs years before completion of the necessary clinical trials. Definitive evidence of the drugs' safety and effectiveness in reducing heart attacks and deaths will only be obtained after such trials are completed. 


\subsection{Atherosclerosis Regression}

To date, the best plausible mechanism to achieve regression of atherosclerosis is by RCT.

In a nutshell, cholesterol, synthesised or deposited in peripheral tissues is returned to the liver by RCT in which HDL plays a central role. HDL is secreted by the liver and intestine in the form of nascent particles consisting of phospholipid and apolipoprotein A1 (Apo A1). Nascent HDL interacts with peripheral cells, such as macrophages, to facilitate the removal of excess free cholesterol (FC), a process facilitated by the ATP-binding cassette protein 1 (ABCA1). FC is generated in part by the hydrolysis of cellular cholesteryl ester (CE) stores by the enzyme cholesterol ester hydrolase (CEH). FC in HDL is then reconverted into CE resulting in mature CE-rich HDL as a result of the plasma cholesterol-esterifying enzyme lecithin: cholesterol acyltransferase (LCAT). Selective uptake of the HDL by the liver is mediated by the scavenger receptor class-B, type I (SR-BI). $\mathrm{CE}$ in the liver derived from HDL contributes to the hepatic-cholesterol pool by processes involving CEH and is used for bile acid synthesis. Cholesterol is eventually excreted from the body or reabsorbed either as its metabolites bile acids or as FC in the bile.

Another, not very publicized, pathway for RCT involves the widely accepted atherogenic lipoproteins and the plasma enzyme CE transfer protein (CETP). CE can be transferred from HDL to Apo B-LPs, such as VLDLs and LDLs, by CETP. Through uptake of LDL by the liver via hepatic LDL receptors, cholesterol can then be transported to the liver, where it contributes to the hepatic-cholesterol pool for excretion or reabsorption.

\subsection{Regression of Atherosclerosis by Stimulation of RCT}

Approaches to obtain regression of atherosclerosis are avidly being investigated. One such approach is the movement of cholesterol from within the body to outside of the body by non-pharmacological methods.

\section{Methods}

\section{Plasma Delipidation}

As early as in the 1970s it was proposed that removing lipid from the plasma by non-pharmacological means, but retaining their protein carriers, the apolipoproteins, in the delipidated plasma may have application to treat hyperlipidemic diseases [4] [5] and in 1978 the importance of apolipoproteins in lipid metabolism was highlighted [6].

By studying and understanding lipid-protein interactions and the interconnectivity of organic solvents with the binding of lipid to proteins, it was considered useful to develop a plasma delipidation process that would result in:

- removal of lipid from plasma without protein denaturation;

- simultaneous extraction of different classes of lipids but at different rates;

- extraction of partial or total lipids from different lipoprotein classes but at different rates.

The rationale of the plasma delipidation system has previously been described [7].

\section{Results}

\subsection{Basic Research}

In 1976 it was first reported that a mixture of butanol and diisopropyl ether (DIPE) attained removal of triglycerides, FC, EC, phospholipids (except gangliosides [7] [8]), and unesterified fatty acids from plasma without protein denaturation. Different lipids were simultaneously extracted, but the rate of extraction was most rapid for unesterified fatty acids, followed by triglycerides, CE, FC and phospholipids at, respectively, decreasing rates [4] [5].

Delipidation of plasma yielded ordered delipidation of HDL, very low-density lipoprotein (VLDL) and LDL in plasma. HDL was more readily delipidated than were LDL and VLDL fractions [4] [5]. Only lipids were removed from the delipidated plasma, all other constituents of plasma including the apolipoproteins remained intact, to the extent that delipidated plasma is used to measure apolipoproteins concentrations [7] [9]. The concentrations and activities of enzymes, including lipid-associated enzymes, were unaffected by the delipidation process [4] [7] [9]-[11]. 


\subsection{Preclinical}

\section{Cell Culture Studies}

Cellular binding activity of LDL and cholesterol-free LDL, obtained by delipidation of plasma with DIPE- butanol, are identical [12]. Delipidated LDL does not stimulate cellular cholesterol ester (ACAT) synthesis, indicating that no cholesterol is presented to those cells by delipidated LDL [12]. Delipidation of plasma converts the spherical HDL particles to disc and amorphous forms but this does not alter receptor-binding activity [12][14].

Delipidated serum, resulting in lipid depletion of all lipoprotein classes, effluxes cholesterol from human mononuclear leukocytes. When delipidated serum substantially depletes the contents of cellular cholesterol, stimulation of intracellular sterol synthesis occurs by a factor of eight to eventually meet the needs of cholesterol for the survival of the cells [15].

However, intracellular sterol synthesis is inhibited by the addition of non-delipidated LDL to the delipidated serum. This indicates that cells preferentially utilize cholesterol given to them rather than synthesizing their own [15]. These studies were confirmed using skin and lung fibroblasts. The activity of HMG-CoA reductase (the rate limiting enzyme for the synthesis of cholesterol) in these cells, increased 5 to 30 fold in media containing delipidated serum. This occurred only after substantial removal of endogenous cellular cholesterol resulting in cellular cholesterol depletion, caused by the delipidated serum [13]. Addition of non-delipidated LDL to the cells resulted in the inhibition of cholesterol synthesis within the cells [14].

It was subsequently established that the component in the delipidated serum responsible for the removal of cholesterol from cells grown in culture was delipidated HDL and that delipidated HDL was a much better (more than 6 fold) cholesterol acceptor than non-delipidated HDL [14].

Thus, delipidated serum contained delipidated LDL that bound to LDL cell receptors but no cholesterol was supplied to these cells. Delipidated serum also contained delipidated HDL that interacted with HDL cell receptors, in this case the modified HDL was capable of removing much more cholesterol from cells compared with HDL particles in non-delipidated serum. It was concluded that delipidated serum supplied less cholesterol to cells and removed more cholesterol from cells.

\subsection{Clinical}

\subsubsection{Batch Type Lipid Apheresis}

A plasma delipidation therapy evolved and was termed lipid apheresis (LA). Initially, batch type extracorporeal lipid apheresis was applied to animals. Twenty five percent of plasma was removed from normolipidemic and diet-induced hyperlipidemic roosters. The plasmas were delipidated and autologously returned back to the same animals.

Different responses were observed when hyperlipidemic animals were compared with normolipidemic animals. In hypercholesterolemic LA-treated animals, an excessive cholesterol pool was rapidly mobilized when compared to normocholesterolemic animals [16].

In the normolipidemic animals the reduction of total cholesterol in plasma was immediate and was significantly sustained for up to 150 minutes. Sixteen hours after LA, the concentrations of total cholesterol in plasma had returned to pre-treatment values. In contrast, with the hypercholesterolemic animals, LA reduced the plasma level of total cholesterol only very transiently. Fifteen minutes after LA, total cholesterol was significantly reduced in the plasma of the hypercholesterolemic animals. Thirty minutes and thereafter, following LA treatment, the concentration of total cholesterol in plasma was generally lower than the pre-treatment value but was not statistically different from the pre-treatment value. Haematological and biochemical parameters in the blood were unaffected by LA treatment [16].

These data strongly suggested that more of an excessive and accessible cholesterol pool in the hypercholesterolemic animals existed that was rapidly mobilizable to the plasma compartment by the LA treatment and supported the cell culture studies.

Virtually immediately after infusion of delipidated plasma, conspicuous changes occurred in HDL. LA markedly induced pre- $\beta$ HDL and at least three other "new" HDL particles in the vascular system and these changes were transient. At approximately 150 minutes after LA, the changes in the HDL pattern had almost returned to the original pre-LA treatment patterns. Concomitant to the changes in the HDL pattern was the immediate reduction in plasma cholesterol concentrations, which was sustained also for 150 minutes [17]. 
Saturated LCAT kinetics was observed for 150 minutes after reinfusion of delipidated plasma in the hypercholesterolemic animals but not normocholesterolemic animals, suggesting that a larger pool of FC was being mobilized in the hypercholesterolemic animals [18].

Cholesterol ester transfer activity (CETA) was twice as high in delipidated hyperlipidemic plasma than in delipidated normolipidemic plasma [19]. This, together with the LCAT kinetic observations, indicated that in the hyperlipidemic state, more CE was being transferred from relipidated HDL to delipidated LDL.

Batch LA-treatments on five consecutive days corresponding to treatments of 1.25 of body plasma volume, resulted in markedly reduced lipid in the arterial wall as well as marked increases in the lumen diameter [20].

Substantive mesenterical adipose tissue was clearly present in the abdomen of diet and hormone induced hyperlipidemic animals. This pool of lipid was drastically diminished when the hyperlipidemic animals were treated by two LA procedures corresponding to LA of 0.5 body plasma volume [21].

\subsubsection{Continuous Lipid Apheresis}

Subsequent to the batch type LA procedure, a continuous LA process was developed in early 1990 [22].

Application of the continuous LA procedure to bovine [23] and porcine [24] normocholesterolemic and diet-induced hypercholesterolemic animals revealed that LA of 1 to 3.5 plasma volumes over 4 to 6 occasions resulted in the following:

- Removal of lipids from plasma by the organic solvent extraction

- Haematological and biochemical parameters were unchanged

- Post-mortem studies showed no adverse effects

- During LA treatment pre-beta and other "new” HDL particles were transiently present in plasma

- LDL was also delipidated

- Rapid, but transient reductions in plasma cholesterol occurred as a consequence of each LA treatment

- After LA much less time was required with the hypercholesterolemic animals for the plasma cholesterol concentration to return to pre-treatment values when compared to normocholesterolemic animals

- Saturated LCAT kinetics occurred in LA treated hypercholesterolemic animals but not normocholesterolemic animals

- CETA was higher in LA treated hyperlipidemic animals than in normolipidemic animals

- Apolipoproteins contents were unaffected by LA treatment

- LA in hyperlipidemic animals mobilized pre-administered radio labelled cholesterol

- Hepatic HMG-CoA reductase activities in the hypercholesterolemic animals were reduced when compared with normocholesterolemic animals

- LA treatment of hypercholesterolemic animals did not result in increases of HMG-CoA reductase activity or plasma lathosterol concentrations

- Histological studies on various segments of arteries indicated removal of lipid from arteries and improvement in morphology of arteries as a consequence of LA treatment

It was interesting that, with these three distinctly different animal models, LA resulted in similar observations.

Figures 1(a)-(c) show the changes in electropherograms, before (A, a; B, 1; C, 1), during, and after LA (A, b-h; B, 2-5; C, 2-6) in the three different models (rooster, bovine and porcine). Obvious, but transient changes in the HDL region were observed. The lipoproteins were stained for lipid. All of the observed "new" HDLs as a consequence of LA, reacted with Apo A1 antibodies.

The LA treatment of the hypercholesterolemic roosters resulted in remarkable reduction of the intensity of lipid in the arterial wall as well as marked increases in lumen diameter.

Figure 2 shows a representative section of a lesion with significant narrowing of the lumen of the thoracic aorta of a hypercholesterolemic rooster and a representative section of a similar site of the thoracic aorta of a hypercholesterolemic animal that had undergone five LA treatments.

Higher magnification of the media sections of the abdominal aorta in hypercholesterolemic roosters showed the presence of intimal accumulation of lipid, whereas, LA treatment of hypercholesterolemic roosters had little such lipid accumulation (Figure 3).

Similar, but less pronounced observations were apparent when hypercholesterolemic bovine and porcine animals were subjected to LA. Histological studies revealed that LA treatments of these hypercholesterolemic animals resulted in removal of lipid from the various arteries studied. 


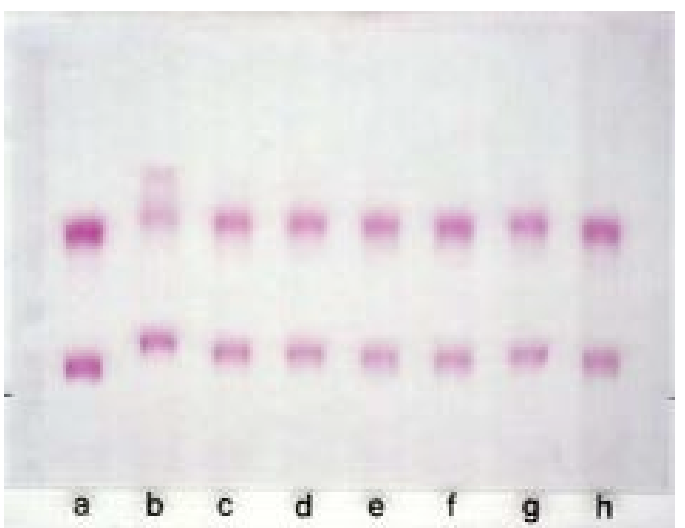

(a)

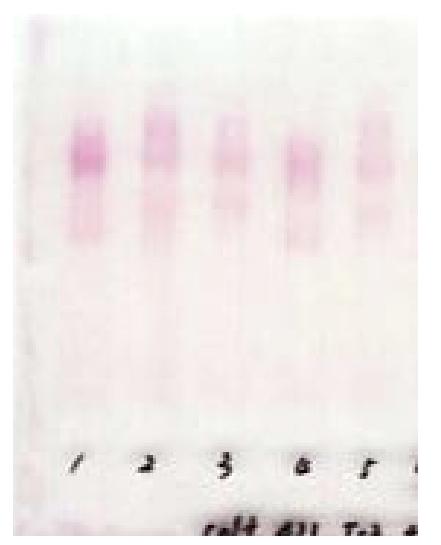

(b)

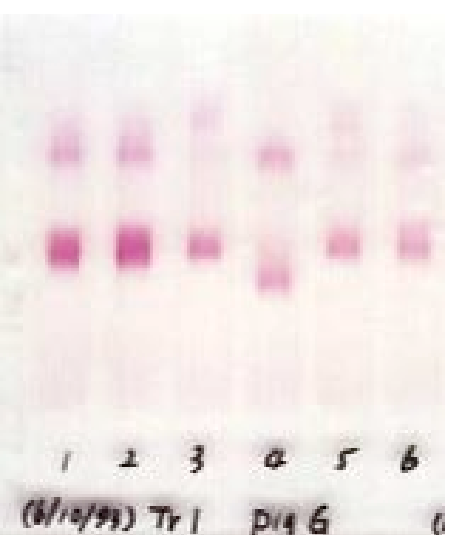

(c)

Figure 1. Representative agarose electropherograms of plasma before and various times after introduction of delipidated autologous plasma into the vascular pool in hypercholesterolemic roosters (a), calves (b) and pigs (c). Lipids in the lipoprotein fractions were stained with Fat Red 7B. Multiple forms of HDLs that reacted to Apo A1 antibodies were observed soon after autologous delipidated plasma was administered intravenously into the animals. These observed changes were transient.

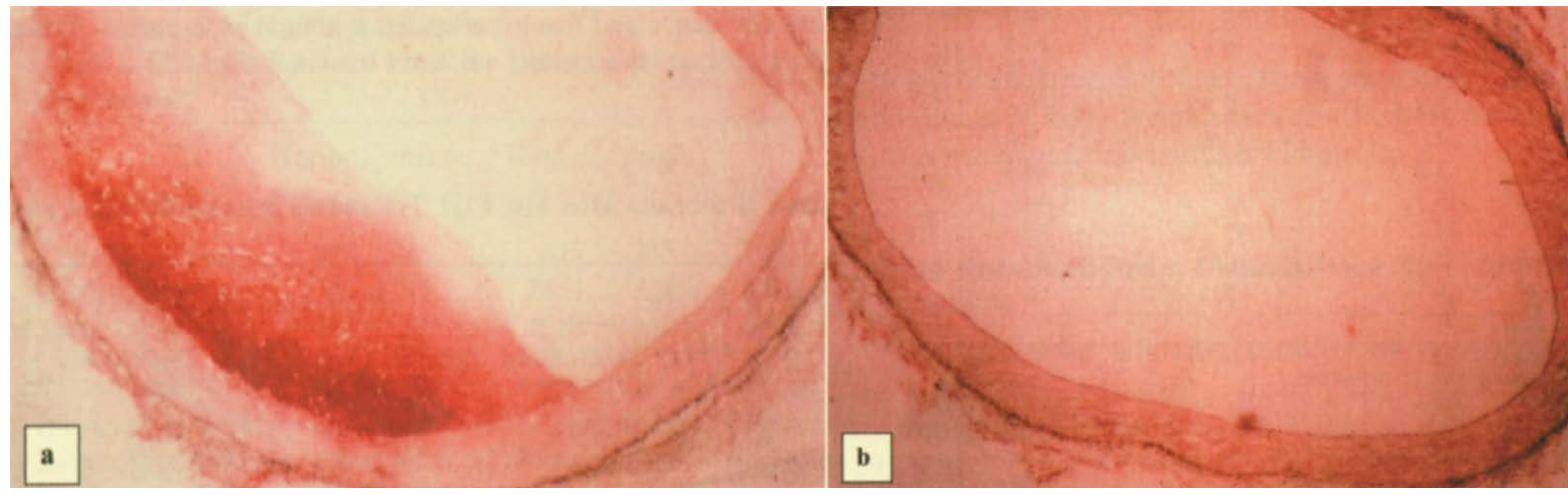

Figure 2. Histological sections (magnified x50) of thoracic aortas stained with Oil Red O. (a) Aorta from hypercholesterolemic rooster showing lipid-rich atherosclerotic plaque and significant luminal narrowing; (b) Aorta from hypercholesterolemic rooster after 5 LA treatments showing no evidence of atherosclerosis.

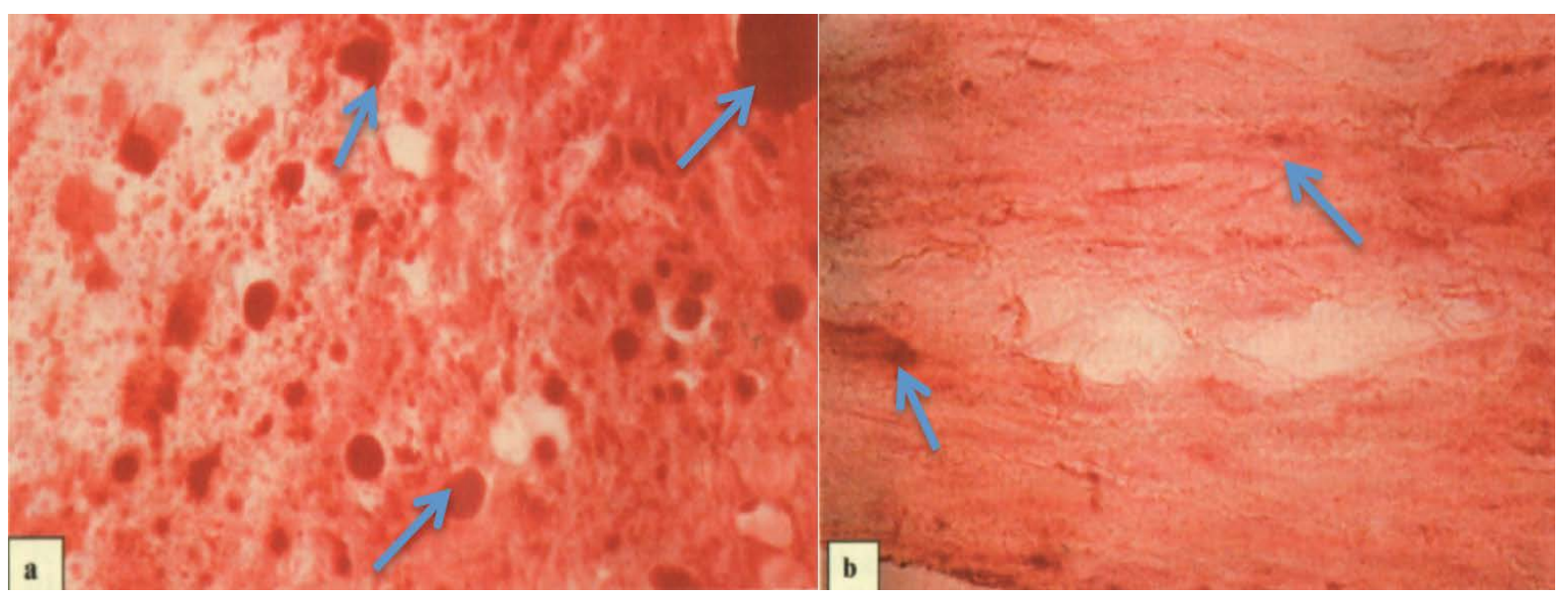

Figure 3. Histological sections (magnified $\times 800$ ) of abdominal aortas stained with Oil Red O. (a) Aorta from hypercholesterolemic rooster showing extensive intimal accumulation of lipid (see arrows); (b) Aorta from hypercholesterolemic rooster after 5 LA treatments showing little lipid accumulation (see arrows). 


\subsubsection{Selective HDL Lipid Apheresis}

Based on the observations reported in the 1970s, that plasma could be delipidated with butanol-ether to yield selective delipidation of HDL; a study was undertaken in non-human primates in the mid 2000s. Twelve weekly LA treatments, corresponding to a total infusion of approximately 4 body plasma volumes of selective HDL delipidated plasma of diet-induced hyperlipidemic African Green Monkeys gave similar results to those obtained with the avian, bovine and porcine models. Again it was shown that LA converted cholesterol-rich HDL particles to cholesterol-poor small, and pre- $\beta$-like HDLs. Pre- $\beta$ HDL had a plasma residence time of $8 \pm 6 \mathrm{~h}$ and was converted entirely to large $\alpha$ HDL, this was within the range of the residence time of pre- $\beta$ HDL observed in the LA treated avian, bovine and porcine models. LA treatment with delipidated plasma reduced diet-induced aortic atherosclerosis in monkeys measured by intravascular ultrasound (IVUS) [25].

In all the animal studies, no serious adverse effects were observed with LA treatments. The promising observations of safety and efficacy achieved by LA treatment with the animal studies led to a randomized, placebocontrolled study to evaluate the safety and feasibility of autologous selective delipidated HDL plasma infusions in human patients with acute coronary syndrome (ACS) [26].

Seven weekly LA treatments of plasma with butanol-ether resulting in selective HDL delipidation, corresponding to a total infusion of approximately two body plasma volumes of selective HDL delipidated plasma, over seven weeks treatment in man, have shown to be feasible and well tolerated in patients with ACS. The levels of the preß-like HDL and $\alpha$ HDL in the delipidated plasma transiently converted from $5.6 \%$ to $79.1 \%$ and $92.8 \%$ to $20.9 \%$, respectively, and confirmed the observations of LA treatment with the avian, bovine, porcine and monkey models. Patients underwent IVUS evaluation of the target vessel during the catheterization of ACS and up to 14 days following the final LA session. The IVUS data demonstrated a numeric trend toward regression in the total atheroma volume of $-12.18 \pm 36.75 \mathrm{~mm}^{3}$ in the delipidated group versus an increase of total atheroma volume of $2.80 \pm 21.25 \mathrm{~mm}^{3}$ in the control group ( $\mathrm{p}=0.268$, N.S.) [26].

The regressions of atherosclerosis although not significant in the humans and monkeys, were similar to the results reported in Apo A1 Milano studies [27].

On the contrary, the regression of atherosclerosis induced by LA in the rooster, bovine and porcine models were significant. These observed differences may be explained by the different precise conditions of the delipidation processes used with the various studies.

With the human and monkey models, the main focus was selective delipidation of HDL in plasma. Whereas, with the rooster, bovine and porcine models, all the lipoprotein classes in the plasma were delipidated. Efficacious mobilization of endogenous lipid stores including regression of atherosclerosis were obtained when plasma delipidation resulted in the removal of over $90 \%$ of cholesterols and triglycerides but retaining over $50 \%$ of phospholipids [7] [9] [16]-[18] [20]-[22]. Regression was also related to the delipidated plasma volume and frequency of LA treatment.

\section{Discussion}

\subsection{Homeostasis versus Non-Homeostasis}

The current accepted concept of increased LDL-C levels being a risk for developing CAD and increased HDL-C levels contributing to the efficiency of RCT are both derived from systems that are at homeostasis in which metabolic equilibria have been achieved.

Lipoproteins carry cholesterol to and from the vessel wall; if too much cholesterol is carried to and too little is carried from the vessel, it accumulates there and causes fatty deposits to build up in the vessel wall.

Conversely, if less cholesterol is carried to and more is removed from the vessel, it depletes fatty deposits from the vessel wall and this is achieved with LA.

During the application of LA, homeostasis is interrupted and a new transient dynamic equilibrium takes place.

Understanding the difference between homeostasis and dynamic transient equilibrium is essential to appreciate how LA results in regression of atherosclerosis.

Relatively high HDL-C plasma levels do not necessarily reflect the efficacy and anti-atherogenecity of RCT. The efficiency of cholesterol flux through RCT pathways is clinically more relevant than the HDL-C concentrations. The concentrations of HDL subclasses, the mobilization of cellular lipids for efflux by delipidated HDL and LDL, and the kinetics of relipidated HDL and LDL metabolism are important determinants of RCT and the risk of atherosclerosis. These dynamic transient equilibrium sequelae regarding the positive effects of stimula- 
tion of CETP to increase RCT are contrary to the understanding of the inhibition concept of CETP to increase HDL-C as is the case with homeostasis.

\subsection{Lipid Apheresis Shows Convincing Promise as a Treatment for Atherosclerosis}

The experimental data of cell culture and clinical studies strongly indicate cholesterol efflux caused by LA is not related to the novo synthesis of cholesterol but is related to mobilization of existing cholesterol stores.

\subsection{Mechanism of Action of Lipid Apheresis Resulting in Regression of Atherosclerosis}

A proposed mechanism of action is summarized in Figure 4 and is based on preclinical and clinical observations.

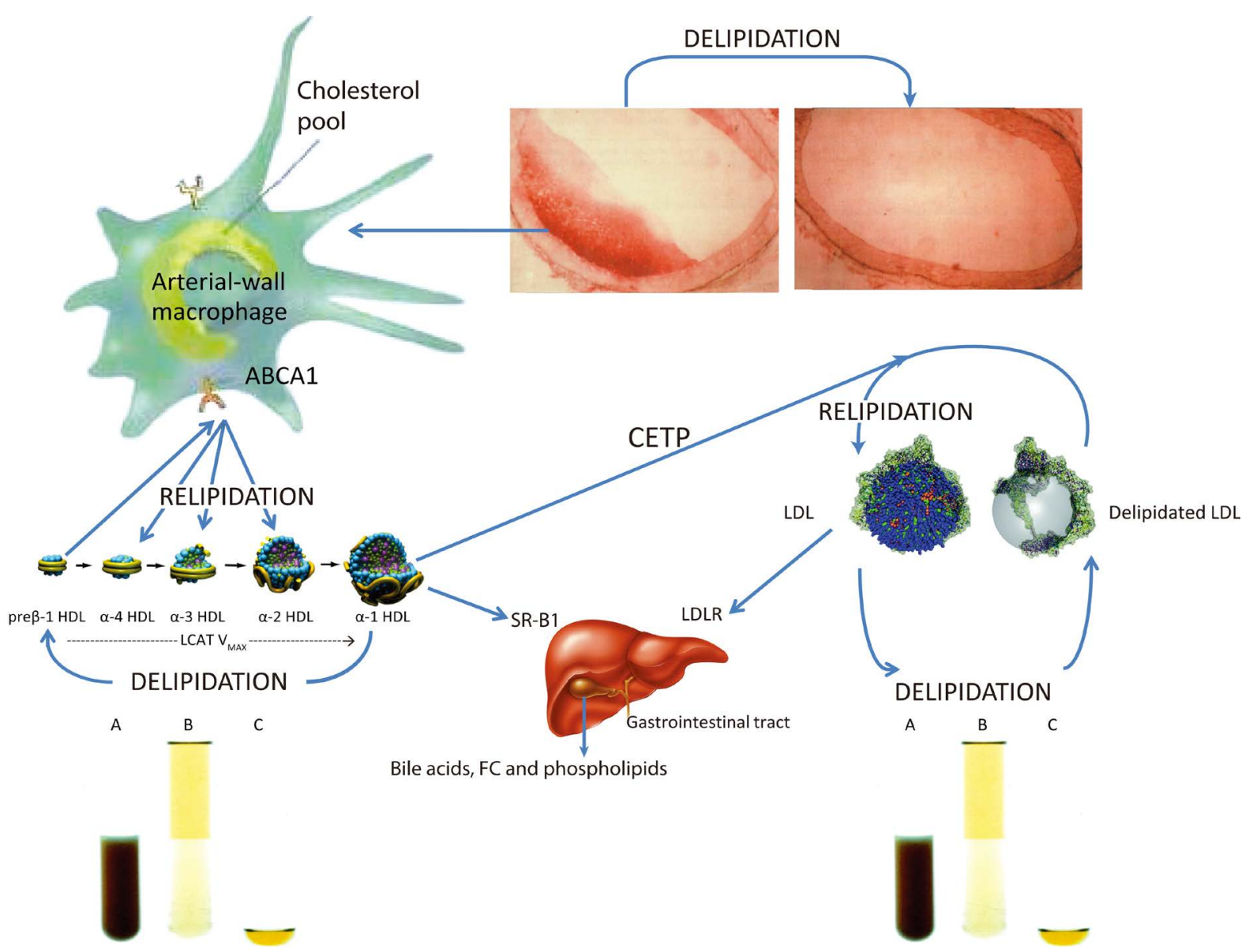

Figure 4. Proposed mechanism of action of LA-induced RCT. Delipidation by LA removes lipid from plasma (test tubes at, 5 and 8 o'clock) (A, undelipidated hyperlipidemic plasma; B, delipidated plasma, the upper organic solvent phase contains the lipids, the lower aqueous phase contains all plasma constituents but no lipids; C, recovered lipids extracted from the hyperlipidemic plasma). $\alpha-1 \mathrm{HDL}$ is converted to pre $\beta-1$ HDL and when administered to the vascular pool, pre $\beta-1$ HDL interacts with ABCA1 receptors of arterial wall macrophages and binds with FC to produce, relipidated $\alpha-4$ HDL, $\alpha-3$ HDL and $\alpha-2$ HDL (9 o'clock). During relipidation of HDL, LCAT activity is at Vmax converting FC to CE in the HDLs (9 o'clock). $\alpha-1$ HDL is transported to the liver where it interacts with SR-B1 (6 o'clock) and its CE content is deposited in the liver. $\alpha-1$ HDL can also transfer its CE to delipidated LDL with the help of stimulated CETP (3 o'clock). Relipidated LDL is transported to the liver and interacts with LDLR and its CE content is deposited in the liver (6 o'clock). The CE in the liver delivered by relipidated HDL and relipidated LDL is converted to FC by the enzyme CEH. FC is partly metabolized to bile acids and both FC and bile acids are secreted through the gastrointestinal tract to the intestine for reabsorption or excretion from the body. HDL = high-density lipoprotein; LDL = low-density lipoprotein; ABCA1 = adenosine triphosphate-binding cassette-A1; LCAT = lecithin cholesterol acyltransferase; CETP = cholesteryl ester transfer protein; LDLR = low-density lipoprotein receptor; SR-B1 = scavenger receptor B1. 
The explanatory notes are indicated by a clock position using the analogy of a 12-hour clock. 5 and 8 o'clock show that plasma delipidation results in the extraction and removal of lipid from plasma (test tubes). As a consequence of plasma delipidation, $\alpha-1$ HDL is converted to preß-1 HDL and when administered to the vascular pool interacts with ABCA1 receptors of arterial wall macrophages and binds with FC to produce relipidated $\alpha-4$ HDL, $\alpha-3$ HDL and $\alpha-2$ HDL (9 o'clock). During this process CEH within the macrophage is activated and converts CE to FC, which binds to the delipidated HDL. Throughout these events LCAT activity works at maximum velocity converting FC to CE within the relipidated HDL in the plasma (9 o'clock). During these processes CE is removed from the macrophage, ultimately ending up in relipidated $\alpha-1 \mathrm{HDL}$.

$\alpha-1$ HDL then has various choices, it can be transported in the vascular system to the liver where it interacts with SR-B1 receptors releasing its cholesterol content to the liver. Liver CEH converts CE to FC, which is partly converted to bile acids. FC and bile acids are secreted through the gastrointestinal tract to the intestine for reabsorption or excretion from the body.

Another choice of relipidated $\alpha-1 \mathrm{HDL}$ is, with the help of CETP, to transfer its CE content to delipidated LDL (3 o'clock). In so doing, delipidated LDL, obtained by delipidation of plasma and administered into the vascular pool, is relipidated. In the course of these events CETP is activated. Relipidated LDL can then be transported to the liver, and interact with LDLR. Consequently the CE content of relipidated LDL may undergo the same fate as CE supplied to the liver by HDL, resulting in excretion of FC or its metabolites, bile acids.

These kinetic and dynamic effects of LA on the body are transient and occur during and temporarily after LA.

Consequently, LA results in reversal of atherosclerosis (12 o'clock).

\section{Conclusions}

RCT is an essential physiologic process that maintains peripheral and total body cholesterol homeostasis. The relationship between RCT and atherosclerosis has long been hypothesised. The molecular mechanisms regulating stimulation of macrophage RCT by plasma delipidation have now been largely elucidated.

The data presented in this report provide insights into the molecular regulation of RCT and indicate that the dynamic rate of macrophage RCT is more strongly associated with atherosclerosis than the steady-state plasma concentration of HDL-C.

Plasma delipidation therapy makes available essential key elements within the body that stimulate RCT resulting in regression of atherosclerosis.

Further work is continuing to establish the optimum delipidation conditions to obtain maximum regression of atherosclerosis, in a manner, that will have wide clinical applications in patients.

\section{References}

[1] Kannel, W.B., Castelli, W.P., Gordon, T. and McNamara, P.M. (1971) Serum Cholesterol, Lipoproteins, and the Risk of Coronary Heart Disease: The Framingham Study. Annals of Internal Medicine, 74, 1-12. http://dx.doi.org/10.7326/0003-4819-74-1-1

[2] Glomset, J.A. and Wright, J.L. (1964) Some Properties of a Cholesterol Esterifying Enzyme in Human Plasma. Biochimica et Biophysica Acta, 89, 266-276. http://dx.doi.org/10.1016/0926-6569(64)90215-9

[3] Glomset, J.A. (1968) The Plasma Lecithins: Cholesterol Acyltransferase Reaction. Journal of Lipid Research, 9, 155167.

[4] Cham, B.E. and Knowles, B.R. (1976) A Solvent System for Delipidation of Plasma or Serum without Protein Precipitation. Journal of Lipid Research, 17, 176-181.

[5] Cham, B.E. and Knowles, B.R. (1976) Changes in Electrophoretic Mobilities of Alpha and Beta Lipoproteins as a Result of Plasma Delipidation. Clinical Chemistry, 22, 305-309.

[6] Cham, B.E. and Knowles, B.R. (1976) In Vitro Partial Relipidation of Apolipoproteins in Plasma. The Journal of Biological Chemistry, 251, 6367-6371.

[7] Cham, B.E. and Chase, T.R. (2013) Intravascular Infusion of Autologous Delipidated Plasma Induces Antiatherogenic Lipoproteins and Causes Regression of Atherosclerosis-Studies in Non-Primates, Monkeys and Humans. Health (Special Issue on Atherosclerosis in Cardiovascular Disease), 5, 19-33.

[8] Wong, C.G. and Ladisch, S. (1983) Retention of Gangliosides in Serum Delipidated by Diisopropyl Ether-Butanol Extraction. Journal of Lipid Research, 24, 666-669.

[9] Cham, B.E. (2007) Manipulation of Reverse Cholesterol Transport System—An Exploration for Rapid Regression of 
Atherosclerosis. Research Journal of Biological Sciences, 2, 291-300.

[10] Groener J.E.M., Van Rozen, A.E. and Erkelens, D.W. (1984) Cholesteryl Ester Transfer Activity. Localization and Role in the Distribution of Cholesteryl Ester among Lipoproteins in Man. Atherosclerosis, 50, 261-271. http://dx.doi.org/10.1016/0021-9150(84)90074-1

[11] Groener, J.E.M., Pelton, R.W. and Kostner, G.M. (1986) Improved Estimation of Cholesteryl Ester Transfer/Exchange Activity in Serum or Plasma. Clinical Chemistry, 32, 283-286.

[12] Innerarity, T.L. and Mahley, R.W. (1978) Enhanced Binding by Cultured Human Fibroblasts of Apo-E-Containing Lipoproteins as Compared with Low Density Lipoproteins. Biochemistry, 17, 1440-1447. http://dx.doi.org/10.1021/bi00601a013

[13] Shakespeare, V. and Postle, A.D. (1979) Regulation of Cholesterol Synthesis in Skin Fibroblasts Derived from Old People. Atherosclerosis, 33, 359-364. http://dx.doi.org/10.1016/0021-9150(79)90187-4

[14] Slater, H.R., Smith, E.B. and Roberston, F.W. (1980) The Effect of Delipidated High-Density Lipoprotein on Human Leukocyte Sterol Synthesis. Atherosclerosis, 35, 41-49. http://dx.doi.org/10.1016/0021-9150(80)90026-X

[15] Slater, H.R. and Robertson, F.W. (1979) A Comparison of Delipidated Sera Used in Studies of Sterol Synthesis by Human Mononuclear Lenkocytes. Journal Lipid Research, 20, 413-416.

[16] Cham, B.E., Kostner, K.M., Dwivedy, A.K., Shafey, T.M., Fang, N.X. and Mahon, M.G. (1995) Lipid Apheresis: An in Vivo Application of Plasma Delipidation with Organic Solvents Resulting in Acute Transient Reduction of Circulating Plasma Lipids in Animals. Journal of Clinical Apheresis, 10, 61-69. http://dx.doi.org/10.1002/jca.2920100202

[17] Cham, B.E., Kostner, K.M., Dwivedy, A.K., Shafey, T.M., Fang, N.X. and Mahon, M.G. (1996) Lipid Apheresis in an Animal Model Causes in Vivo Changes in Lipoprotein Electrophoretic Patterns. Journal of Clinical Apheresis, 11, 61-70. http://dx.doi.org/10.1002/(SICI)1098-1101(1996)11:2<61::AID-JCA2>3.0.CO;2-8

[18] Kostner, K.M., Smith, J.L., Dwivedy, A.K., Shafey, T.M., et al. (1997) Lecithin Cholesterol Acyltransferase Activity in Normocholesterolemic and Hypercholesterolemic Roosters: Modulation by Lipid Apheresis. European Journal of Clinical Investigation, 27, 212-218. http://dx.doi.org/10.1046/j.1365-2362.1997.960643.x

[19] Groener, J.E.M., Van Rozen, A.E. and Erkelens, D.W. (1984) Cholesteryl Ester Transfer Activity: Localization and Role in the Distribution of Cholesteryl Ester among Lipoproteins in Man. Atherosclerosis, 50, 261-271. http://dx.doi.org/10.1016/0021-9150(84)90074-1

[20] Cham, B.E., Kostner, K.M., Shafey, T.M., Smith, J.L. and Colquhoun, D.M. (2005) Plasma Delipidation Process Induces Rapid Regression of Atherosclerosis and Mobilization of Adipose Tissue. Journal of Clinical Apheresis, 20, 143-153. http://dx.doi.org/10.1002/jca.20060

[21] Cham, B.E. and Smith, J.L. (1994) Lipid Apheresis in an Animal Model Causes Acute Reduction in Plasma Lipid Concentrations and Mobilization of Lipid from Liver and Aorta. Pharmacology Life Science Advances, 13, 25-32.

[22] Cham, B.E. (1990) Autologous Plasma Delipidation Using a Continuous Flow System. US Patent No.: 4895558A.

[23] Cham, B.E., Kostner, K.M., Colquhoun, D.M. and Dwivedy, A.K. (1994) Lipid-Apheresis: An in Vivo Application of Delipidation but Apo Lipoprotein Retention in Plasma of Calves by a Continuous Extracorporeal Procedure. Atherosclerosis, 109, 158. http://dx.doi.org/10.1016/0021-9150(94)93644-7

[24] Cham, B.E., Kostner, K.M., Colquhoun, D.M. and Dwivedy, A.K. (1994) Lipid-Apheresis: An in Vivo Application of Delipidation but Apo Lipoprotein Retention in Plasma of Pigs by a Continuous Extracorporeal Procedure. Atherosclerosis, 109,159. http://dx.doi.org/10.1016/0021-9150(94)93645-5

[25] Sacks, F.M., Rudel, L.L., Conner, A., Keefe, A., Kostner, G., Baki, T., et al. (2009) Selective Delipidation of Plasma HDL Enhances Reverse Cholesterol Transport in Vivo. Journal of Lipid Research, 50, 894-907. http://dx.doi.org/10.1194/jlr.M800622-JLR200

[26] Waksman, R., Torguson, R., Kent, K.M., Pichard, A.D., Suddath, W.O., Satler, L.F., et al. (2010) A First-in-Man, Randomized, Placebo-Controlled Study to Evaluate the Safety and Feasibility of Autologous Delipidated High-Density Lipoprotein Plasma Infusions in Patients with Acute Coronary Syndrome. Journal of the American College Cardiology, 55, 2727-2735. http://dx.doi.org/10.1016/j.jacc.2009.12.067

[27] Nissen, S.E., Tsunoda, T., Tuzcu, E.M., Schoenhagen, P., et al. (2003) Effect of Recombinant Apo A-I Milano on Coronary Atherosclerosis in Patients with Acute Coronary Syndromes. A Randomized Controlled Trial. JAMA, 290, 2292-2300. http://dx.doi.org/10.1001/jama.290.17.2292 